\title{
Quantum states in proteins and protein assemblies: The essence of life?
}

\author{
STUART HAMEROFF ${ }^{\mathrm{a}}$, JACK TUSZYNSKI ${ }^{\mathrm{b}}$ \\ ${ }^{a}$ Departments of Anesthesiology and Psychology, Center for Consciousness Studies, \\ The University of Arizona Health Sciences Center, Tucson, Arizona USA 85724; \\ ${ }^{\mathrm{b}}$ Department of Physics, University of Alberta, Edmonton, T6G 2J1, Canada
}

\begin{abstract}
Activities in living cells are performed by protein conformational dynamics which in turn are governed by quantum mechanical van der Waals London forces in intra-protein "hydrophobic" pockets. In assemblies of proteins with periodic lattice geometry such as cytoskeletal actin and microtubules (as well as ordered water on their surfaces), Bose-Einstein condensation, quantum coherent superposition and quantum computation with entanglement may occur as a collective effect of these forces due to metabolic coherent phonon pumping. Decoherence can be avoided through isolation/shielding by actin gelation, Debye layer screening and water/ion ordering and topological quantum error correction. As an example, quantum spin transfer through organic molecules is more efficient at higher temperatures than at absolute zero. The unitary oneness and ineffability of living systems may depend on mesoscopic/macroscopic quantum states in protoplasm.
\end{abstract}

Keywords: Bose Einstein condensation, cytoskeleton, decoherence, evolution, life, London forces, microtubules, protein conformation, quantum coherence, quantum superposition

\section{OIL VERSUS WATER: HYDROPHOBIC POCKETS IN PROTEINS}

All chemistry including biochemistry is based on quantum interactions, so living systems-like non-living systemsdepend on quantum states at the level of chemical bonds. However the unitary oneness and ineffability of living systems have suggested that higher level quantum properties such as Bose-Einstein condensation, quantum coherent superposition and entanglement may operate in biology. ${ }^{1-3}$ But quantum effects are apparently washed out at scales larger than individual atoms or sub-atomic particles, at warm temperatures, and in aqueous media. Thus the possibility of quantum states playing functional roles at mesoscopic or macroscopic scales in "warm, wet and noisy" biological systems seems unlikely due to environmental decoherence. On the other hand evolution may have solved the decoherence problem so that mesoscopic/macroscopic quantum states are essential features of biological systems. ${ }^{4}$

Living cells are $80 \%$ water, and many polar, water soluble biomolecules play key roles in cell function. However there are also extensive non-polar "oily" regions in living cells which exclude water. These are called hydrophobic regions, and they occur within proteins", lipid membranes and nucleic acids (e.g. the "pi stack" of DNA). If organized quantum states exist in cells they are presumably integrated among hydrophobic regions of various cellular components and organelles. However here we consider only proteins and geometric arrays of proteins (i.e. protein assemblies) in cytoplasm, the bulk protoplasm between cell membrane and nucleus.

Proteins are folded chains of amino acids strung together by peptide bonds (Figure 1, top). Each of the twenty different amino acids which comprise proteins have distinct side groups with varying degrees of polarity and water solubility. Amino acids with side groups (or "residues") which are non-polar and insoluble in water ("hydrophobic") include phenylalanine, leucine, isoleucine, tryptophan, tyrosine, valine and histidine (in decreasing order of hydrophobicity). The side groups in phenylalanine, tryptophan and tyrosine ("aromatic" amino acids) are benzene-like (or "indole", in the case of tryptophan) carbon ring structures with resonant electron orbitals. (Histidine has a five sided ring with three carbons and two nitrogens.) The side groups in leucine, isoleucine and valine have several methyl groups, each of which is a

${ }^{\mathrm{a}}$ hameroff@u.arizona.edu; phone 525626 5605; www.consciousness.arizona.edu/hameroff
jus@phys.ualberta.ca; phone 780492 3579; $\underline{\text { http://www.phys.ualberta.ca/ jtus }}$ 

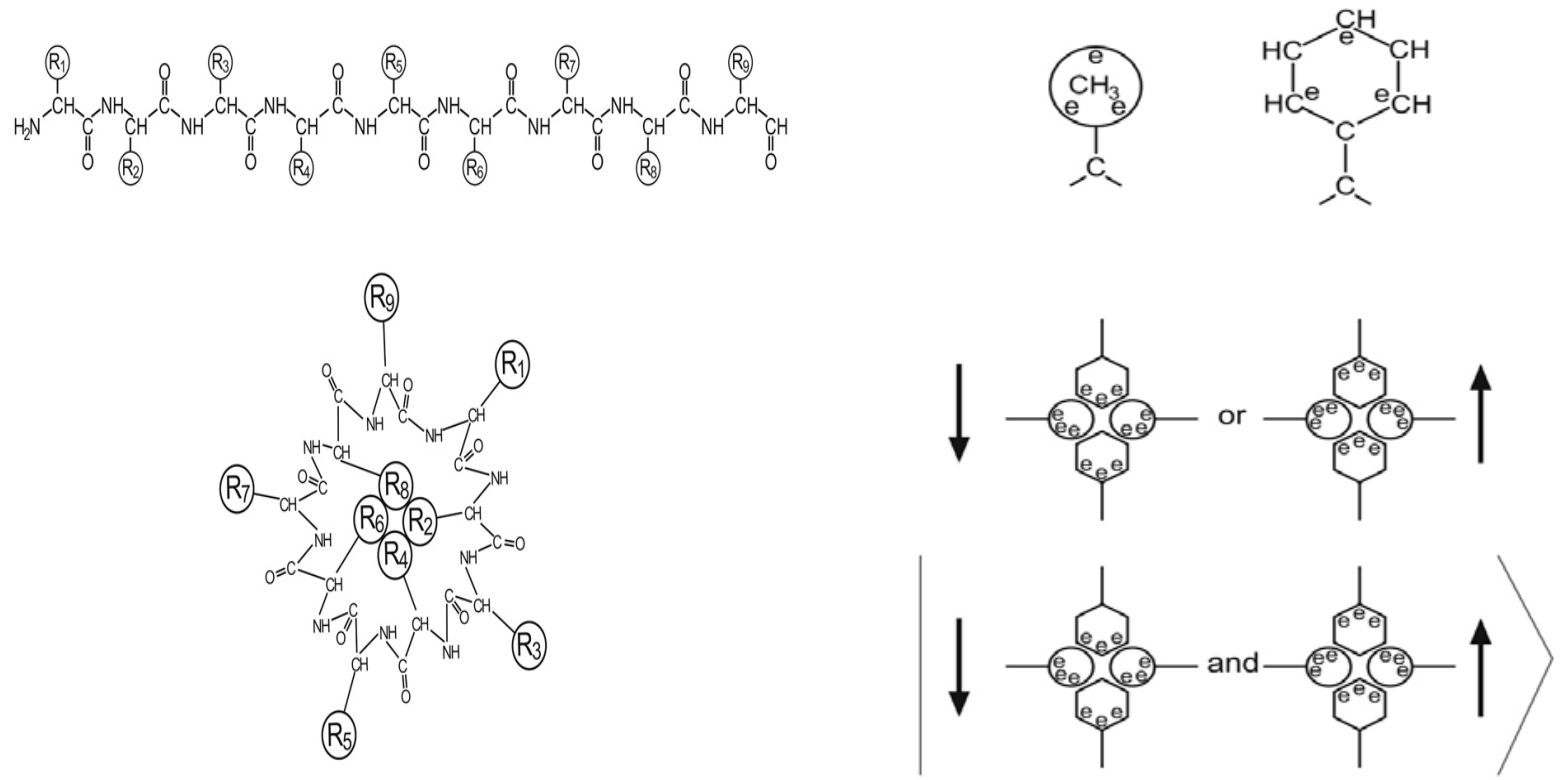

Figure 1. Left: Top: amino acids link together by peptide bonds. R1-9 are side groups of varying polarity. Bottom: hydrophobic side groups (R2, R4, R6, R8) avoid polar liquid environment and bury together inwardly to form hydrophobic pocket. Polar sidegroups $(\mathrm{R} 1, \mathrm{R} 3, \mathrm{R} 5, \mathrm{R} 7, \mathrm{R} 9)$ stay in contact with aqueous environment as do polar carbonyl $\mathrm{C}=\mathrm{O}$ groups. Right:Top: On left is methyl group with 3 shared mobile electrons, on right is aromatic ring with 3 shared mobile electrons. Middle: 2 methyl and 2 aromatic groups form hydrophobic pocket, attracted by van der Waals London forces/instantaneous net dipoles. Bottom: Quantum superposition of London forces, dipoles in both directions.

carbon with three hydrogens. Both aromatic and methyl groups are non-polar, but have electron clouds of shared, delocalizable electrons and are thus "polarizable" - dipoles can be induced in the electron clouds. The non-hydrophobic amino acids have side groups which are charged, or polar, and thus soluble in water. ${ }^{6}$

As proteins fold into their functional shapes, hydrophobic groups avoid the liquid environment and bury themselves together in the protein interior, forming hydrophobic pockets, not unlike oil droplets forming in, or on the surface of water (Figure 1 Left, bottom) It is, in fact, the hydrophobic effect and attractions among non-polar hydrophobic groups by van der Waals forces which drive protein folding. Although the confluence of hydrophobic side groups are small, roughly $1 / 30$ to $1 / 250$ of protein volumes, they exert enormous influence in the regulation of protein dynamics and function. Several hydrophobic pockets may work cooperatively in a single protein (Figure 2, Left). Hydrophobic pockets may be considered the "brain" or nervous system of each protein.

\section{LONDON FORCES AND PROTEIN CONFORMATIONAL DYNAMICS}

The currency of real-time cellular function is conformational dynamics of proteins. Enzymatic function, intra-cellular and cellular movement, signaling, transport and information processing depend on changes in protein shape. In some cases proteins require biochemical energy to change shape; in other cases binding of various ligands, voltage changes or influence of neighboring molecules are sufficient. For example membrane ion channels can change shape in response to binding of neurotransmitters or changes in voltage to become permeable to ion flow, allowing depolarization waves to travel along membranes. Intracellular proteins including cytoskeletal structures change shape to facilitate signaling and rearrangement of cellular architecture. However the regulation of protein conformation is poorly understood. 

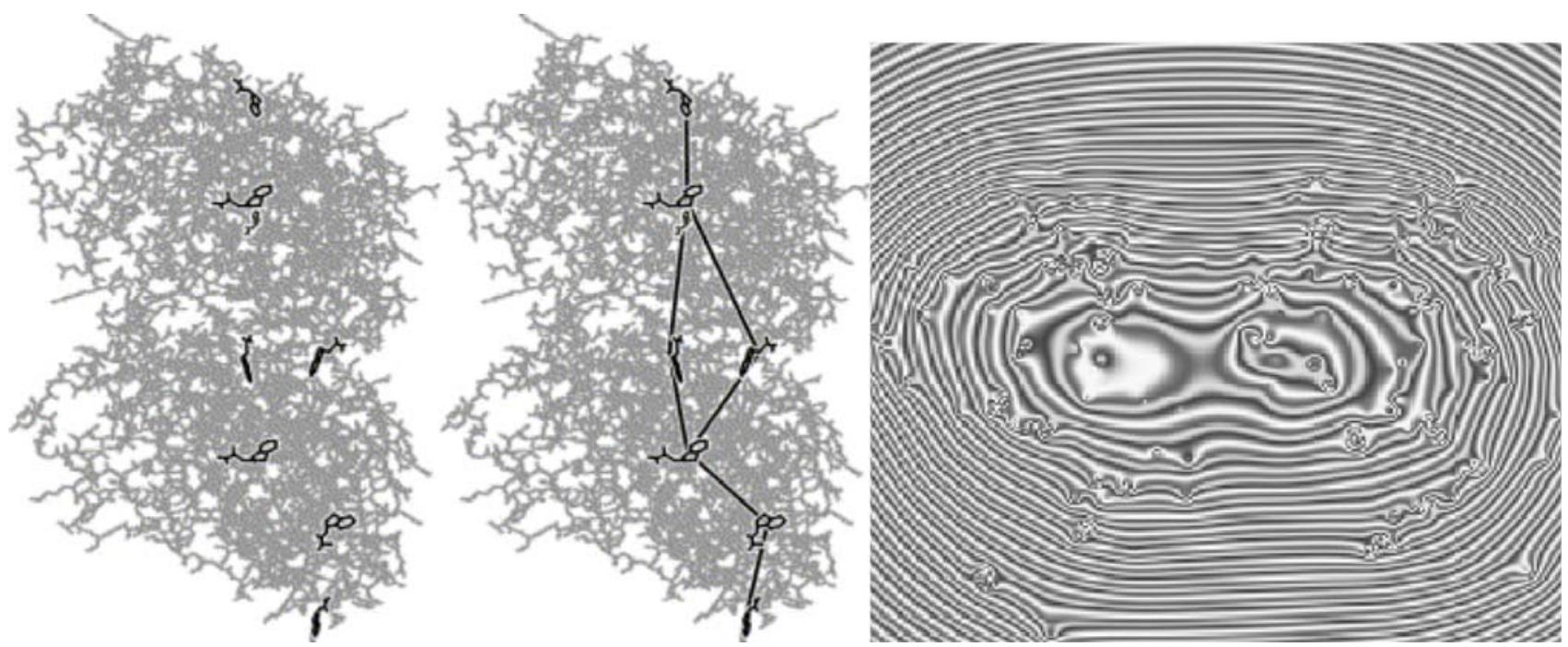

Figure 2. Left: Representation of atomic structure of tubulin, the subunit protein of microtubules (without C-termini) ${ }^{7,8}$. Locations of aromatic, hydrophobic tryptophan amino acid side groups are shown, indicating several hydrophobic pockets in which London dipoles may act cooperatively to regulate conformation Right: Electrostatic map of a horizontal slice through tubulin near the "neck" between the alpha and beta monomers (Figure 2 Left) shows a "double well" of positive charge. ${ }^{11}$

Proteins change shape over a number of time scales. ${ }^{9}$ Small parts of proteins, for example protrusions from the main body of the protein change as fast as femtoseconds $\left(10^{-15} \mathrm{sec}\right)$. Other conformational changes are very slow. The binding of oxygen by blood hemoglobin changes the shape of hemoglobin until the oxygen is released, a process that may take many seconds or minutes. Most functional conformational transitions in which the entire protein changes shape globally occur in the time scale of nanoseconds $\left(10^{-9} \mathrm{sec}\right)$ to 10 picosecond $\left(10^{-11} \mathrm{sec}\right)$. An ion channel, for example, may change its shape in a nanosecond or so, but remain open for longer, long enough for ions to flow through.

Interactions among side groups within proteins provide a large amount of energy, e.g. thousands of kiloJoules per mole $\left(\mathrm{kJ} \mathrm{mol}^{-1}\right)$. Yet proteins are only marginally stable against denaturation by an excess of $40 \mathrm{~kJ} \mathrm{~mol}^{-1}$, and switching and conformational changes in proteins may be triggered by very small amounts of energy. For example open and closed states of the potassium channel differ by only small amounts of energy, regulated by a hydrophobic lever. ${ }^{10}$ How do such high energy systems change shape due to extremely low energy stimuli?

The answer seems to be that high energy interactions from chemical and ionic bonding among charged, polar side groups tend to cancel out, such that protein conformation is a "delicate balance among powerful countervailing forces". ${ }^{6}$ More dynamic, low energy interactions such as the forces active in non-polar hydrophobic pockets are thus leveraged or amplified to regulate conformation. These are the van der Waals forces.

Van der Waals forces occur between dipoles, and include three types:

1) Permanent dipole - permanent dipole

2) Permanent dipole - induced dipole

3) Induced dipole - induced dipole

Type 3 induced dipole - induced dipole interactions are the weakest but most purely non-polar. They are known as London dispersion forces, and although quite delicate (40 times weaker than hydrogen bonds) are numerous and influential. The London force attraction between any two atoms is usually less than a few kiloJoules, however thousands occur in each protein, and they may act collectively. As other forces cancel out, London forces in hydrophobic pockets can govern protein conformational states.

London forces ensue from the fact that atoms and molecules which are electrically neutral and structurally symmetrical nevertheless have instantaneous electric dipoles due to asymmetry in their electron distribution. The electric field from 

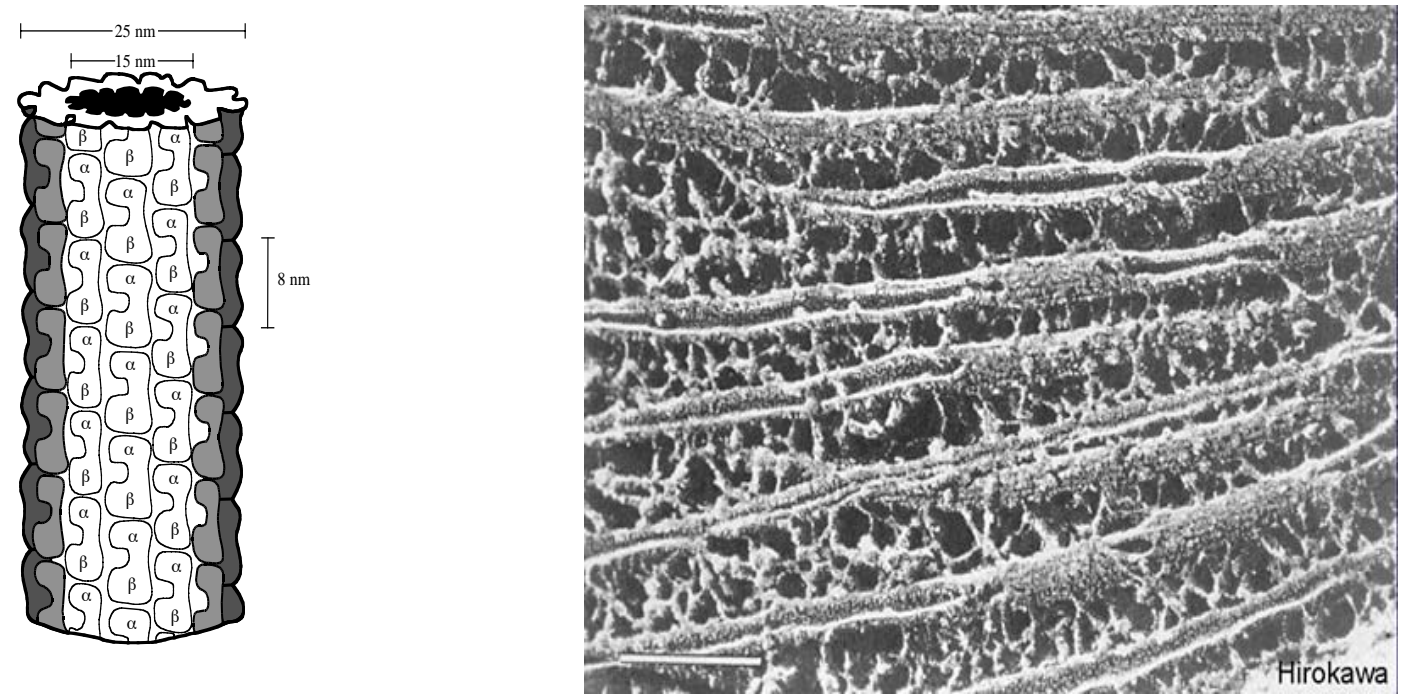

Figure 3. Left: Microtubules are hollow cylindrical polymers of tubulin proteins, each a "dimer" of alpha and beta monomers. The outer core diameter is $25 \mathrm{~nm}$. Right: Immunoelectron micrograph of microtubules in neuronal dendrite cytoplasm interconnected by microtubule associated proteins. Scale bar (lower left): $100 \mathrm{~nm}$. From Hirokawa ${ }^{13}$ with permission.

each fluctuating dipole induces and couples to others in electron clouds of adjacent non-polar amino acid side groups. Due to inherent uncertainty in electron location, instantaneous London forces are quantum mechanical effects (Figure 1).

London forces have important biological implications. Anesthetic gases act to ablate consciousness in hydrophobic pockets of certain brain proteins. ${ }^{5}$ The anesthetic gas molecules do so by forming their own van der Waals London interactions with non-polar amino acid groups in the pockets, presumably impairing normally occurring London force effects necessary for protein functions required for consciousness.

How are movements of electrons, even collective movements of many electrons, able to tip the "delicate balance of countervailing forces" in proteins to cause conformational changes in the range of nanoseconds to ten picoseconds? Due to the Mossbauer effect, electronic motions should couple to nuclear motions (and thus conformation) via a recoil phenomenon. ${ }^{11}$ However the nuclear/conformational movement would be slight. Because of the small mass of electrons relative to that of protons and neutrons, a one nanometer shift of a single electron would move the nuclear mass, and hence protein conformation by only $10^{-8}$ nanometers, the diameter of a single carbon atom nucleus. However the charge on each electron is equivalent to that on protons, and collective electron charge movements by London forces are thus able to influence nuclear motion and protein conformation. ${ }^{12}$

London forces are quantum effects; the instantaneous dipoles are actually probabilistic densities of electron clouds. If the electron dipoles couple to protein conformation, then proteins should exist in quantum superposition of different conformations, albeit of very small separations. For example the Mossbauer shift of $10^{-8}$ nanometers would mean that the protein's nuclei, and thus the protein, would separate "from itself" by this distance, the diameter of a carbon atom nucleus. But even this slight separation coupled cooperatively among atomic nuclei throughout the protein may tip "the balance between countervailing forces". So a protein in superposition of two locations which differ by $10^{-8}$ nanometers, may collapse/reduce/decohere into two distinct conformations which differ in location from each other by several angstroms. Proteins may thus be considered to act as quantum bits, or "qubits" (Figure 8).

Some evidence supports quantum effects in hydrophobic pockets playing functional roles in determining protein conformation. Roitberg et $\mathrm{al}^{14}$ showed functional protein vibrations which depend on quantum effects centered in two hydrophobic phenylalanine side groups, and Tejada et $\mathrm{al}^{15}$ have evidence to suggest quantum coherent states exist in the protein ferritin. Nonlocal quantum interactions ${ }^{16}$ and quantum tunneling appear to guide protein folding. ${ }^{17}$ Such effects are confined to individual proteins, and the role of the environment must be considered. 

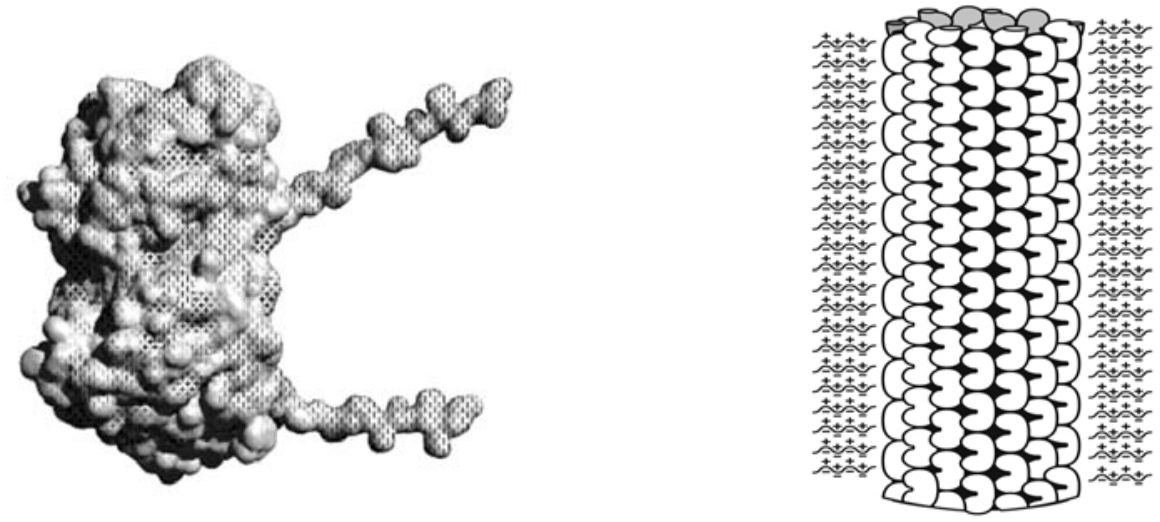

Figure 4. Left: A map of the electric charge distribution on the surface of a tubulin dimer with C-termini tails. ${ }^{14,15}$ Right: Microtubule with negatively charged C-termini which attract positively charged ions, forming a Debye double layer which may serve to screen/shield quantum states in microtubules from decoherence. ${ }^{4,11}$

Polar amino acid side groups which interface with the protein's external milieu may allow environmental effects to dominate, relegating the protein to a "slave". ${ }^{18}$ Random thermal motion in an aqueous environment would promote decoherence and prevent or disrupt quantum states. However structured, cooperative environments may allow collective effects promoting large scale quantum coherence of proteins.

\section{CYTOPLASMIC GEL AND THE CYTOSKELETON}

Living material is termed protoplasm which includes cell membranes and cell interiors. Cell interiors excluding the nucleus are comprised of organelles embedded in cytoplasm, which is composed mainly of water and networks/matrices of proteins collectively termed the cytoskeleton. There are three main components of the cytoskeleton: actin, intermediate filaments and microtubules, all organized around a central focus called the centrosome. Situated just outside and adjacent to the cell nucleus, the centrosome is composed of a curious organelle called the centriole (Figures 7 and 8 ) embedded in an electron-rich protein medium.

Actin protein is the most dynamic cytoskeletal structure, rapidly polymerizing and depolymerizing, and by so doing changing the character of cytoplasm from a liquid phase ("sol", i.e. solution) when actin is disassembled, to a solid gelatinous phase ("gel") when actin is assembled/polymerized. ${ }^{19}$ Such "sol-gel transitions" which can occur as rapidly as 40 times per second account for intracellular flow of cytoplasm (e.g. "amoeboid movement). Because actin surfaces are negatively charged, in the gel phase nearly all polar cell water molecules are bound and "ordered". Thus, during the gel phase, the view of cell interiors as a "minestrone soup" of floating organelles is incorrect. Rather, cytoplasmic gel is a quasi-solid state medium.

Intermediate filaments are fairly stable, and their precise function is poorly understood. On the other hand microtubules can be either stable, or dynamic, and play many essential roles in cell biology. In asymmetrical cells such as neurons (with long extensions called axons and dendrites), microtubules play structural roles, as more or less permanent girders (Figure 3, Right). During cell division, or "mitosis", microtubules depolymerize and then repolymerize as mitotic spindles, separating "daughter chromosomes" and establishing the architecture for daughter cells. The microtubule spindles pull the daughter chromosomes toward two poles anchored by centrioles which seem to guide navigation and cytoplasmic organization. Each centriole is a pair of barrel-like structures arranged curiously in perpendicular tandem, 

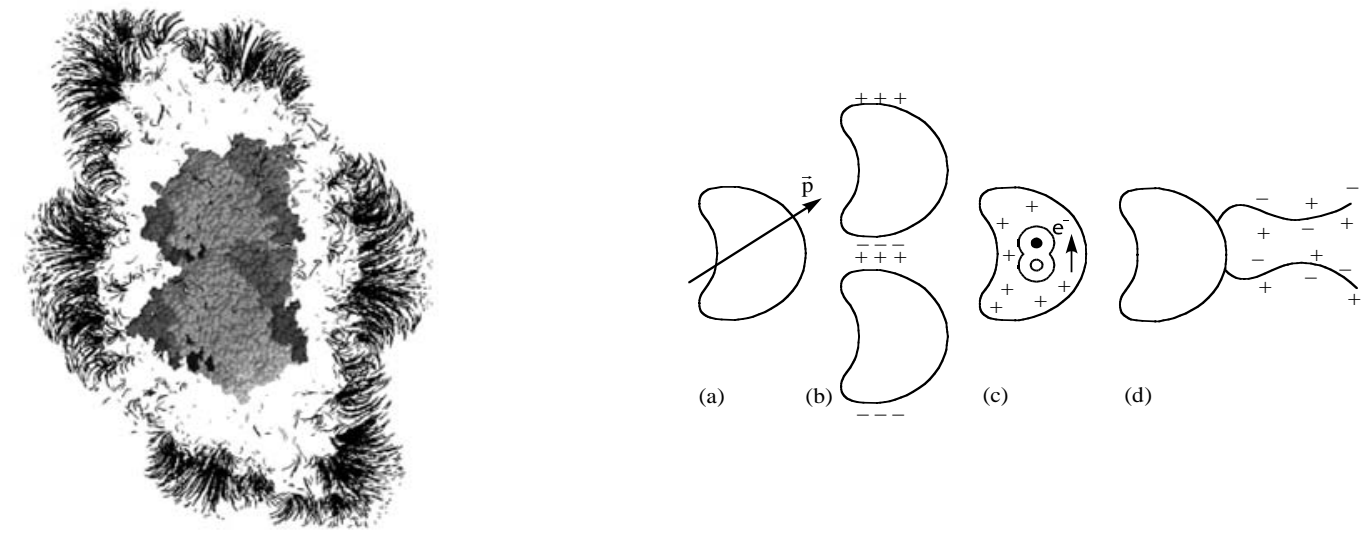

Figure 5. Left: A view of the attractive regions about a tubulin dimer as would be experienced by another dimer. The smallest principal moment of inertia of the dimers is perpendicular to the page, the middle one is aligned vertically, the largest principal moment horizontally. ${ }^{11}$ Right: The various contributions to the dipole moment of a tubulin dimer in a microtubule: (a) the intrinsic dipole moment of the protein, ${ }^{11}$ (b) the double layer formed when neighboring tubulins are bound in a protofilament (Figure 5, Left), (c) possible internal dipole from London force transitions in hydrophobic pocket or double well (Figure 3, Right), (d) double layer caused by counter ions (Figure 5 , Right). ${ }^{11}$

and (like mitotic spindles) are comprised of microtubules (Figures 6 and 7). There are a number of questions regarding mitosis, but one compelling issue is how the processes are coordinated in space and time by centrioles to generate a geometric spindle structure that maintains itself at steady state. A review in Science concluded: "Robustness of spindle assembly must come from guidance of ....microtubules by a field". ${ }^{20}$

Microtubules are cylindrical polymers of the protein tubulin and are 25 nanometers (nm) in diameter (Figure 3). ${ }^{21}$ The cylinder walls of microtubules are comprised of 13 longitudinal protofilaments which are each a series of tubulin subunits. Each tubulin subunit is an $8 \mathrm{~nm}$ by $4 \mathrm{~nm}$ by $5 \mathrm{~nm}$ heterodimer which consists of two slightly different classes of $4 \mathrm{~nm}, 55,000$ dalton monomers known as alpha and beta tubulin. The tubulin dimer subunits within the cylinder walls are arranged in a hexagonal lattice which is slightly twisted, resulting in differing neighbor relationships among each subunit and its six nearest neighbors. Pathways along neighbor tubulins form helices which repeat on any protofilament every 3, 5, 8, 13 etc. rows (the "Fibonacci series").

Each tubulin has a surplus of negative surface charges, with a majority on the alpha monomer; thus each tubulin is a permanent dipole (beta plus, alpha minus). Consequently microtubules can be considered "electrets": oriented assemblies of dipoles which are predicted to have piezoelectric, ferroelectric and spin glass properties. ${ }^{22}$ In addition, negatively charged C-termini "tails" extend outward from each monomer, attracting positive ions from the cytoplasm and forming a plasma-like "Debye layer" surrounding the microtubule (Figures 4 and 5).,11

Biochemical energy is provided to microtubules in several ways: tubulin-bound GTP is hydrolyzed to GDP in microtubules, and microtubule associated proteins which attach at specific points on the microtubule lattice are phosphorylated. In addition microtubules may possibly utilize nonspecific thermal energy for "laser-like" coherent pumping, for example in the GigaHz range by a mechanism of "pumped phonons" suggested by Fröhlich. ${ }^{23-25}$ Simulation of coherent phonons in microtubules suggest that phonon maxima correspond with functional binding sites of microtubule associated proteins. ${ }^{26}$

In centrioles (as well as cilia, flagella, basal bodies etc.) microtubules fuse into doublets or triplets. Nine doublets or triplets then form larger barrel-like cylinders which in some cases have internal structures connecting the 

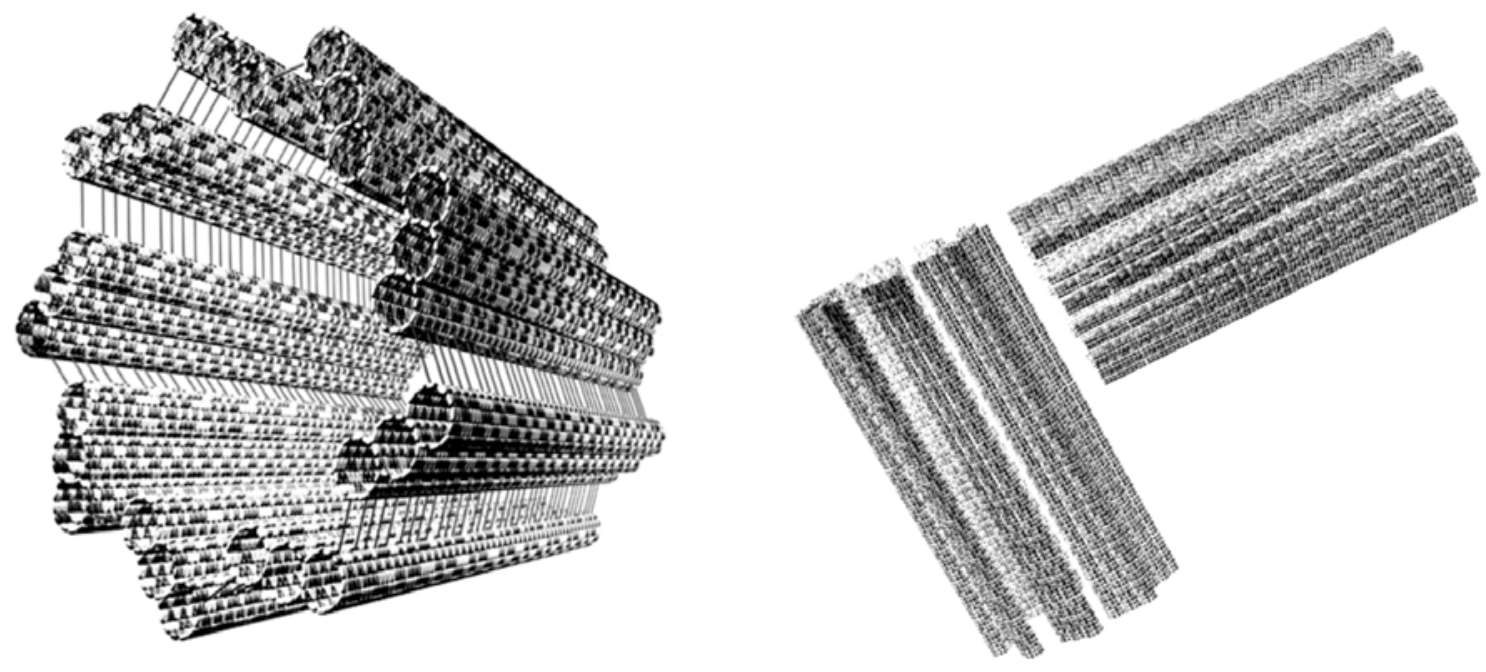

Figure 6. Left: Centriole cylinder (one half of a centriole) is comprised of 9 microtubule triplets in a skewed parallel arrangement. The cylinder inner core is approximately 140 nanometers in diameter and the cylinder is 750 nanometers in length. Right: A centriole is comprised of two such cylinders arranged in perpendicular tandem.
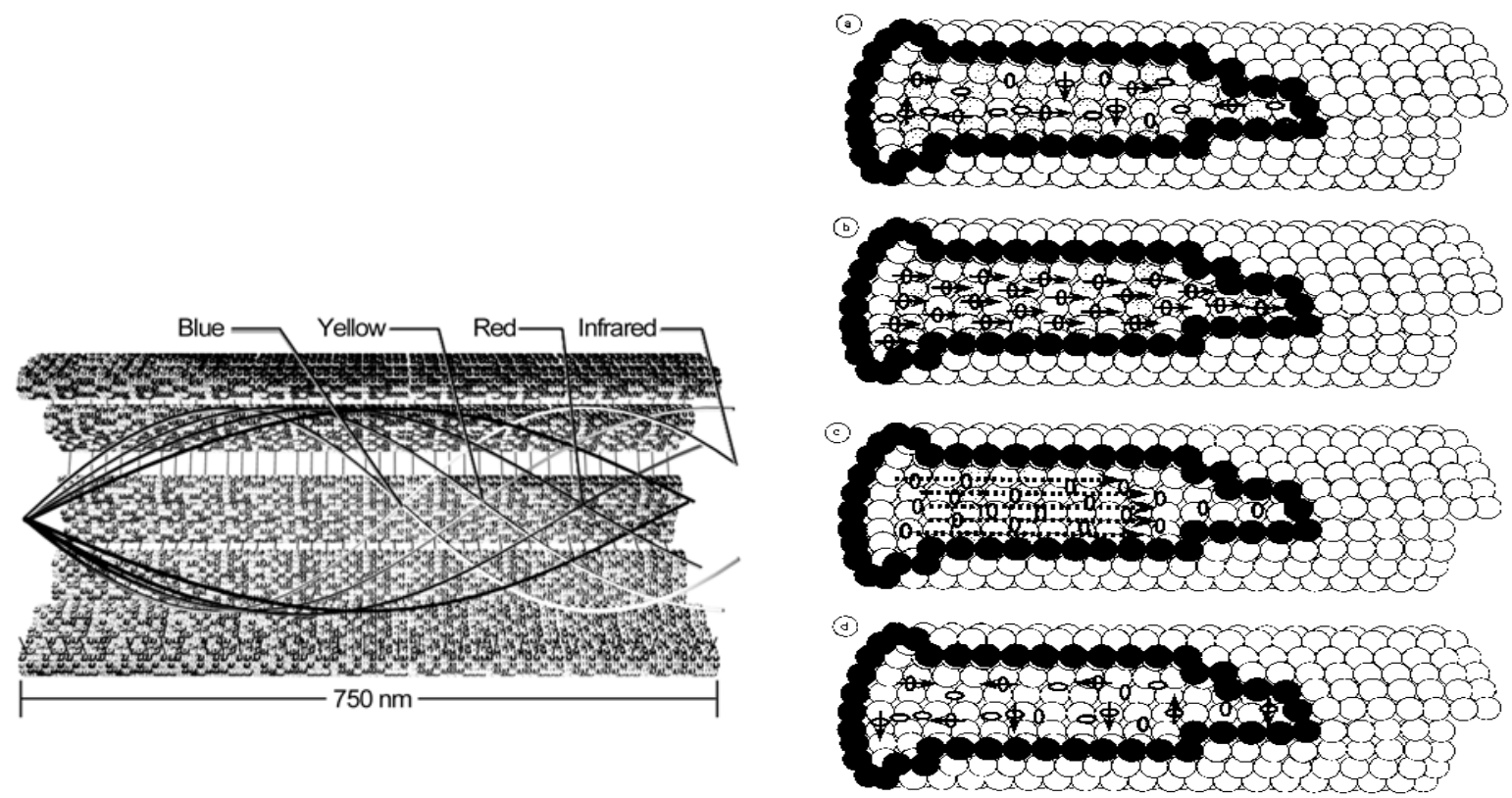

Figure 7. Left: Cutaway view of centriole cylinder showing wavelengths of visible and infra-red light suggesting possible waveguide behavior. ${ }^{28}$ Right: A schematic representation of the process of superradiance in a single microtubule. ${ }^{29}$ Each oval signifies a water molecule in the lowest rotational energy state. Each oval with an arrow is a water molecule in the first excited rotational state. The process is cyclic (a, b, c, d, a, b...). A) Initial state: Energy gain due to thermal fluctuations of tubulins increases the number of water molecules in the first excited rotational energy state. B) A collective mode of the system of water molecules in rotationally excited states. A long-range coherence is achieved inside a microtubule by means of spontaneous symmetry breaking. C) A collective mode of the system of water molecules in rotationally excited states loses its energy collectively, and creates coherent photons in the quantized electromagnetic field. D) Water molecules, having lost their first excited rotational energies by superradiance, start again to gain energy from the thermal fluctuation of tubulins, and the system of water molecules recover the initial state (a). From Jibu et al. ${ }^{29}$ 
doublets/triplets. Two cylinders in perpendicular tandem form the centriole which initiates and organizes mitosis (cell division) and other cell movements.

Centrioles and cilia are involved in photoreceptor functions in single cell organisms and primitive visual systems. ${ }^{27}$ Cilia are also found in all retinal rod and cone cells. The dimensions of centrioles and cilia are equivalent to wavelengths of visible and infrared light, and waveguide functions have been suggested (Figure 7). Because mitosis (cell division) is organized by centrioles and requires precise, mirror-like acivities, quantum entanglement mediated by quantum optical effects between centrioles has been proposed. ${ }^{28}$

\section{FRÖHLICH COHERENCE, QUANTUM FIELD THEORY AND MICROTUBULES}

To account for communication and organization in living systems, physicist Herbert Fröhlich ${ }^{23-25}$ suggested that a membrane or large chain of macromolecules possessing longitudinal dipole vibrations could act like a Bose-Einstein condensate. Fröhlich's proposal was based on the notion that biological systems are far from equilibrium, and have considerable amounts of available energy which lead to non-linear behaviors. The concept that many body systems far from equilibrium and governed by nonlinear kinetic laws may display macroscopic self-organized order is consistent with approaches such as Prigogine's dissipative structures and Haken's synergetics. ${ }^{30}$

In Fröhlich's model several modes of dipole vibrations are excited by a continuous supply of metabolic energy. The electric vibrations couple to acoustic vibrations inherent in the structure through non-linear dynamics; Fröhlich specified dipoles in hydrophobic regions of proteins as the relevant dipoles. If the supplied energy is sufficiently large compared to the energy lost, the system attains a stationary state in which the dipole vibrations channel into a single longitudinal mode (the one with minimum frequency). The common mode increases its population enormously in a way reminiscent of Bose-Einstein condensation. The single mode, which can exhibit long range correlations is thus akin to laser-like coherent pumped phonons in the range of $10^{9}$ to $10^{11} \mathrm{~Hz}$. The proposed frequency is in the microwave range, ${ }^{31}$ and corresponds to recognized protein conformational transitions in the range of nanoseconds to 10 picoseconds. Fröhlich's proposal may be seen in a context of quantum field theory whose proponents have viewed living matter as a sea of electric dipoles organized by electret states, and the consequent ordering of water around biomolecules, providing collective states of ordered symmetry. ${ }^{32,33}$

In a typical nonbiological system, component dipoles are random and disordered, resulting in an overall symmetry ("rotationally invariant"). In living systems, order is induced by rotational alignment along filamentous electrets such as microtubules and other cytoskeletal structures. According to quantum field theory and the "Goldstone theorem", symmetry breaking ("Bose condensation") results in long range interactions among system components (dipoles) conveyed by massless particle/waves ("Goldstone bosons"). The energy required to generate massless bosons is invested in the electret states of biomolecules and correlated fluctuations of their surrounding water and ions. For example Celaschi and Mascarenhas ${ }^{34}$ showed that electret activation energy of biomolecules (0.2-0.4 electron volts) is equivalent to the hydrolysis of one ATP or GTP molecule (and what Davydov predicted for initiation of solitons ${ }^{40}$ ). Consequently solitons, massless bosons, and Fröhlich's coherent polarization waves may be synonymous.

Del Giudice and colleagues ${ }^{35-37}$ concluded that electromagnetic energy penetrating into cytoplasm would be confined inside filaments whose diameters depended on the original symmetry breaking ("Bose condensation") of ordered dipoles. Any electric disturbance produced by thermally fluctuating dipoles or by any other source would be confined inside filamentous regions. Ordering is preserved outside the filaments and is disrupted only inside where energy becomes concentrated (the "Meissner effect"). The diameter of the self focusing energy filaments depends on the polarization density, or ordering of biological water. Del Guidice's group calculated a self focusing diameter of about 15 nanometers, precisely the inner diameter of microtubule and concluded the cytoskeleton is the material consequence of dynamic self focusing of polarization waves in the cytoplasm. The observed diameters of self focused optical beams in simple nonbiological liquids are of the order of microns; correlation among components is created by propagation of waves rather than as a specific property of the material itself. Thus focusing occurs in cytoplasm of eukaryotic cells due to the spatial coherence and ordering imparted by cytoskeletal electret behavior. 
Along similar lines, Mari Jibu, Kunio Yasue and Scott Hagan ${ }^{29,38}$ calculated that Fröhlich dynamics of ordered water on microtubule surfaces, particularly the internal hollow core of microtubules, would result in quantum optical modes termed super-radiance and self-induced transparency (Figure 7 Right). Evanescent quantum photons in the infra-red region would propagate losslessly, in an optical analogue to superconductivity. The quantum coherent state would involve a region of 50 microns, allowing quantum coherence to spread through tissues.

Another relevant approach is through the zero point energy of the quantum vacuum. Virtual particle/waves (photons) in the quantum vacuum "pop into and out of existence", giving rose to a description of spacetime as the "quantum foam". Zero point energy may be measured through the Casimir effect, in which e.g. two parallel plates are held close together such that the narrow gap excludes photons whose wavelength is larger than the gap. The relative surplus of photons outside the gap exerts a force pushing the plates together. Experiments with parallel plates placed 0.6 to 6.0 microns apart confirm the Casimir effect, finding a very small, but measurable force pushing the plates together. ${ }^{39}$

Physicist George Hall ${ }^{40}$ calculated the Casimir force on a microtubule whose cavity diameter (" $d$ ") is 15 nanometers, roughly 100 times smaller than the gap $d$ in the described experiment. As the force depends on $\mathrm{d}^{-4}$ the Casimir effect would be much higher, a million times greater (neglecting effects of the cylindrical surface compared to the planar surface). Depending on length, Hall calculated a Casimir pressure of 0.5 to 20 atmospheres on a microtubule.

\section{QUANTUM COMPUTATION IN MICROTUBULES}

In a specific proposal for a mechanism of consciousness, Penrose and Hameroff ${ }^{41-44}$ have suggested that microtubules function as quantum computers, with tubulin proteins within microtubules acting as quantum bits, or qubits. The model (Orchestrated objective reduction, Orch OR) is summarized here:

- Conformational states of individual tubulins within neuronal microtubules are determined by quantum mechanical London forces within the tubulin interiors which can induce conformational quantum superposition.

- While in superposition, tubulins communicate/compute with entangled tubulins in the same microtubule, and in other microtubules in the same neuron, and in microtubules in neighboring neurons, and through macroscopic regions of brain via tunneling through gap junctions and possibly tunneling nanotubes.

- Quantum states of tubulin/microtubuless are isolated/protected from environmental decoherence by biological mechanisms which include phases of actin gelation, ordered water, plasma-like Debye layering, coherent pumping and topological quantum error correction .

⿷ Microtubule quantum computations/superpositions are tuned or 'orchestrated' by microtubule associated proteins during a classical, liquid phase which alternates (e.g. at $40 \mathrm{~Hz}$ ) with a quantum, solid state phase of actin gelation.

⿷ Following periods of pre-conscious quantum computation (e.g. on the order of tens to hundreds of milliseconds) tubulin superpositions reduce or collapse by Penrose quantum gravity 'objective reduction' (OR). The classical output states which result from the OR process are chosen non-algorithmically ('non-computably') and govern neural events by binding of microtubule associated proteins, regulating synapses and membrane functions etc.

E The reduction or 'self-collapse' in the orchestrated objective reduction 'Orch OR' model is suggested to be a 'conscious moment', linked to Penrose's quantum gravity mechanism which ties the process to fundamental spacetime geometry. This connection enables a pan-protopsychist approach to the `hard problem' of subjective experience.

This implies that tubulins can exist in quantum superposition of two or more possible states until threshold for quantum state reduction (i.e. by Penrose quantum gravity mediated "objective reduction") is reached. The tubulin superposition is suggested to involve separation of tubulin "from itself" by a Fermi length, the diameter of an atomic (i.e. carbon) nucleus. Despite the slight separation distance, the two states presumably evolve to functional conformations which differ in location by angstroms. 

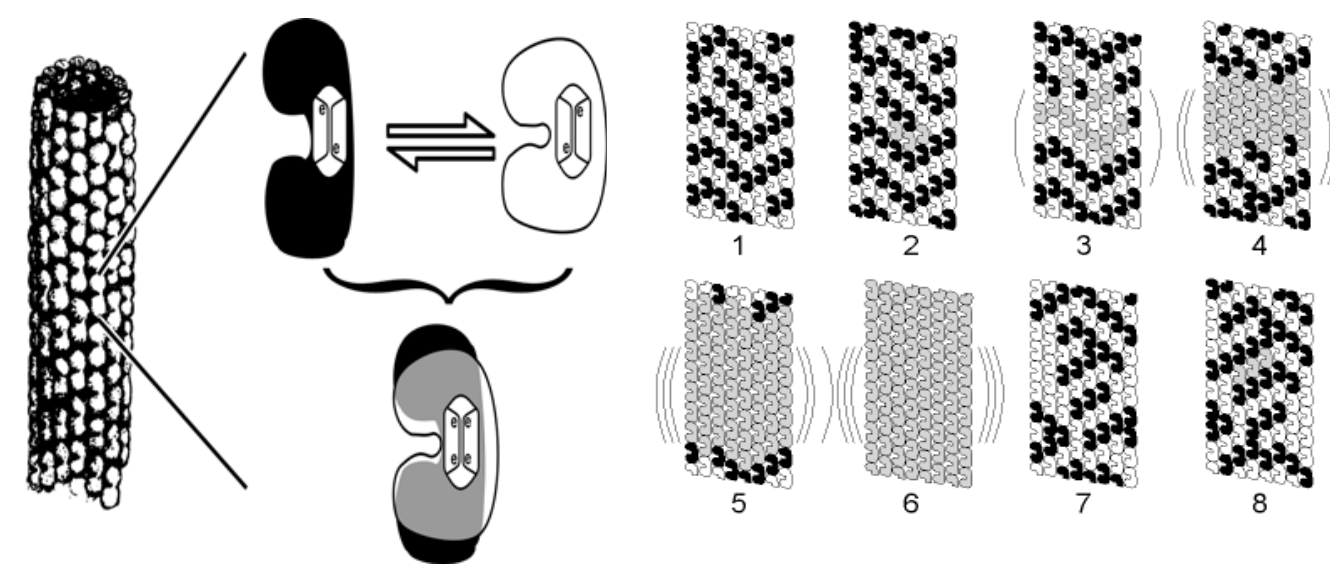

Figure 8. Left: Microtubule (MT) structure: a hollow tube of 25 nanometers diameter, consisting of 13 columns of tubulin dimers arranged in a skewed hexagonal lattice. Right top): Each tubulin molecule may switch between two (or more) conformations, coupled to London forces in a hydrophobic pocket. Right (bottom): Each tubulin can also exist in quantum superposition of both conformational states. Right: Microtubule automaton sequence simulation in which classical computing (step 1) leads to emergence of quantum coherent superposition (steps 2 - 6) in certain (gray) tubulins. Step 6 (in coherence with other microtubule tubulins) meets critical threshold related to quantum gravity for self collapse (Orch OR). Consciousness (Orch OR) occurs in the step 6 to 7 transition. Step 7 represents the eigenstate of mass distribution of the collapse which evolves by classical computing automata to regulate neural function. Quantum coherent superposition begins to re-emerge in step 8.

Not surprisingly the Penrose-Hameroff Orch OR model has engendered criticism because of the apparent and obvious problem of decoherence. Cytoplasm is considered a hostile environment for mesoscopic or macroscopic quantum states because it is viewed as "warm, wet and noisy", all of which would presumably lead to decoherence of quantum states. Let's consider these objections:

"Warm": Quantum information technologies are being constructed at absolute zero temperatures to avoid thermal decoherence. By those standards biological systems are sizzling hot, for example humans function at around 37.6 degrees centigrade. However some quantum states like lasers do function at warm temperatures, and are energetically pumped to produce quantum coherence. In the Fröhlich approach, thermal energy is used in a similar fashion to pump coherence in lattices of dipoles. Microtubules and other cytoskeletal lattice structures are ideal for such a mechanism. Moreover quantum spin transfer between quantum dots connected by benzene rings (the same structures found in aromatic hydrophobic amino acids) is more efficient at warm temperature than at absolute zero. ${ }^{45}$ The authors conjecture that the "flexibility" of the resonant benzene electrons is advantageous to quantum processes by harnessing ambient thermal energy.

"Wet": Cytoplasm is often viewed as an aqueous medium in view of its water content of $80 \%$. However when actin is polymerized, cell water is apparently completely ordered resulting in a quasi solid state medium. ${ }^{19}$ Moreover ordered water at cytoskeletal surfaces would also be driven by Fröhlich coherence pumped by heat and biochemical energy. The sum total of hydrophobic space in interiors of proteins, membranes and nucleic acids provides a non-polar, spatially distributed region throughout living systems.

"Noisy": It is assumed that the aqueous, thermal cytoplasmic environment is noisy. However if coherent pumping and ordered water occur, noise may not be an issue. In the brain, apparent noise in terms of electrically recorded membrane fluctuations have been found to have long range correlations, and thus may not be noise but some underlying coupling. ${ }^{47}$

Attempting to disprove the possibility of quantum computation in brain microtubules, University of Pennsylvania physicist Max Tegmark ${ }^{48}$ calculated that microtubule quantum states at physiological temperature would decohere a 
trillion times too fast for physiological effects, with a calculated decoherence time of $10^{-13}$ seconds. Neurons generally function in the range of roughly 10 to 100 milliseconds, or $10^{-2}$ to $10^{-1}$ seconds.

However Tegmark didn't actually address specifics of the Penrose-Hameroff Orch OR model, nor any previous theory, but rather proposed his own quantum microtubule model which he did indeed successfully disprove. For example Tegmark assumed quantum superposition of a soliton wave traveling along a microtubule, "separated from itself" by 24 nanometers. The Penrose-Hameroff model actually proposed quantum superposition of tubulin proteins separated from themselves by the diameter of their atomic nuclei. This discrepancy alone accounts for a difference of 7 orders of magnitude in the decoherence calculation. Moreover Tegmark did not consider other factors included in the PenroseHameroff proposal such as plasma phase screening, actin gel isolation, coherent pumping and quantum error correction topology intrinsic to microtubule geometry.

Hagan et $\mathrm{al}^{4}$ used Tegmark's own decoherence formula and, correcting for the stipulations in the Penrose-Hameroff model, calculated microtubule decoherence times in the range of $10^{-2}$ to $10^{-1}$ seconds or longer (without benefit of topological quantum error correction). Those results were published in Physical Reviews E, the same journal in which Tegmark's original article was published.

Technological quantum computation became more feasible with the advent of quantum error correction codes in the early 1990s. ${ }^{49}$ This means that algorithms run on the quantum computer can detect and correct errors due to random localized decoherence before they destroy the global quantum state. In some cases specific topological structural geometry of the quantum computer enhances the error correction. For example toroidal surfaces may have global, topological degrees of freedom which are protected from local errors and decoherence. ${ }^{50}$

The topology of microtubule lattices involves helical windings which repeat on any given protofilament according to the Fibonacci series $(3,5,8,13,21 \ldots)$. Each of the winding patterns also corresponds with pathways along specific aromatic amino acid groups which may promote exciton or spin transfer along those pathways (Figure 9). ${ }^{51}$ If the winding patterns, rather than individual tubulin subunits are taken as qubits, then microtubules may be resistant to decoherence by virtue of topological quantum error correction. If any individual tubulin in a winding decoheres, it will resonate back into quantum superposition by other tubulins. In this scenario the output of the quantum computation will be specific combinations of winding patterns which are known to induce binding of microtubule associated proteins which in turn determine cytoskeletal structure and function. This may also be seen to utilize the Aharonov-Bohm effect in which alternate loop pathways may exist in quantum superposition.

Recent quantum information technology utilizes unpaired electron quantum spin (rather than electron location) to represent information ("spintronics"). As previously mentioned, electron quantum spin transfer through organic benzene molecules (the same as found in aromatic amino acids) is more efficient at room temperature than at absolute zero. ${ }^{45}$ At physiological temperature, unpaired electron spins form networks in protein interiors, ${ }^{52}$ and nonlocal quantum effects are important in protein folding. ${ }^{14}$

What about microtubules and spintronics? Microtubules are ferromagnetic lattices which align parallel to strong magnetic fields, which may be accounted for by single unpaired electrons per tubulin. Atomic structure of tubulin shows two positively charged areas $(\sim 100-150 \mathrm{meV})$ near the alpha-beta dimer "neck" separated by a negatively charged area of about 1.5 nanometers (Figure 2). ${ }^{11}$ This region constitutes a double well potential which should enable inter-well quantum tunneling of single electrons and spin states since the energy depth is significantly above thermal fluctuations ( $\mathrm{kT}=25 \mathrm{meV}$ at room temperature). The intra-tubulin dielectric constant is only 2, compared to roughly 80 outside the microtubule. Hence neither environmental nor thermal effects threaten quantum spin states in the double well. 

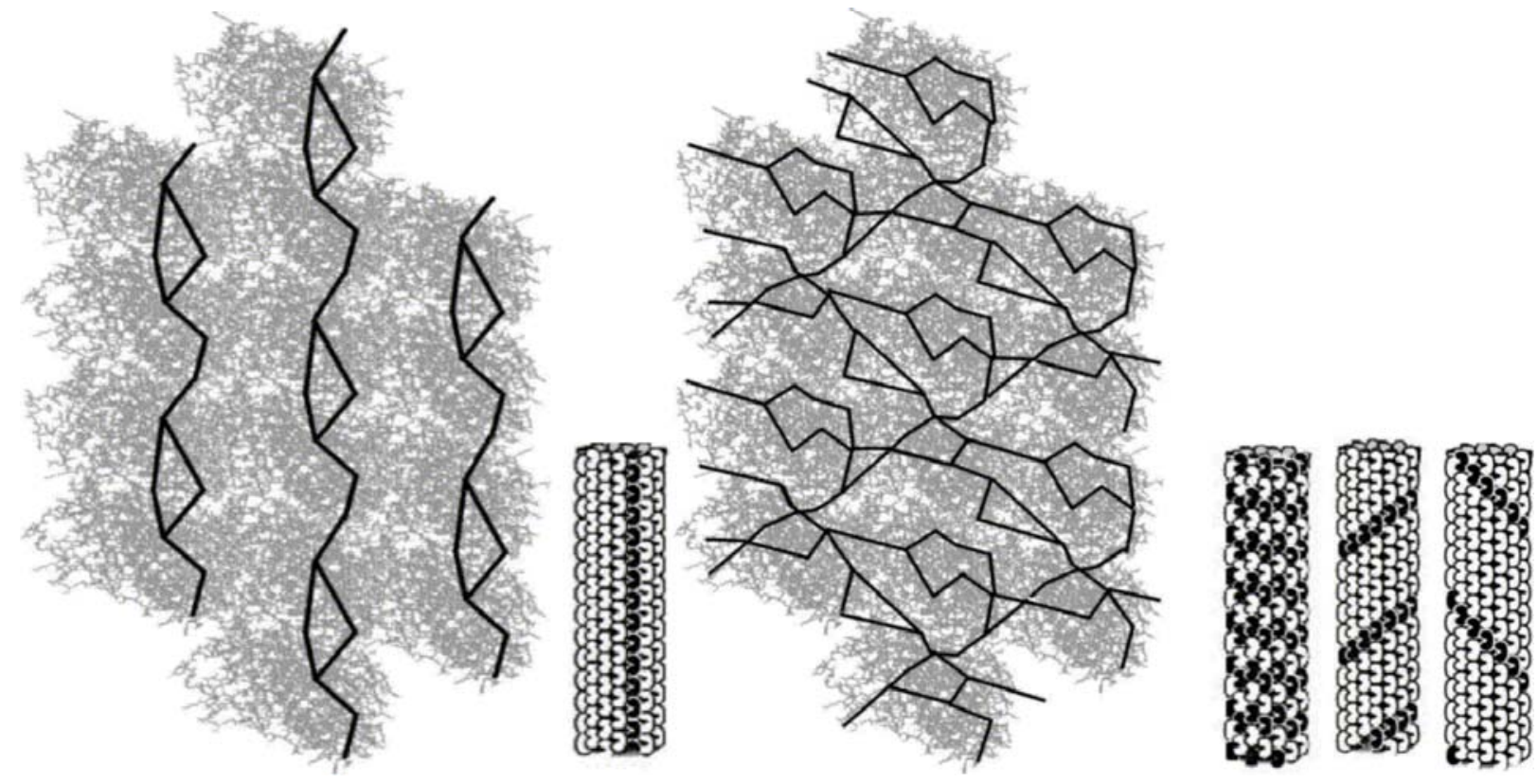

Figure 9. Left: A lattice neighborhood of 7 tubulin dimers showing connected locations of tryptophans and vertical pathways along protofilament; Right: Lattice neighborhood of 7 tubulin dimers with connected locations of aromatic amino acids phenylalanine and histidine which correspond with winding patterns along the 3,5 and 8 helical starts. Alternate pathways could provide topological quantum error correction via the Aharonov-Bohm effect.

\section{CONCLUSION: WHAT IS LIFE?}

We have discussed a number of possible implementations of organized quantum states and processes in cytoplasm, suggesting that such states and processes are relevant to the essential features of living systems.

What is life? Life is a process generally described in terms of its properties and functions such as self-organization, metabolism (energy utilization), adaptive behavior, reproduction, and evolution. Whether or not this functional description is complete is a matter of contention. Two broad types of approaches have attempted to characterize the essential nature of the life process: (1) functionalism and (2) vitalism.

Functionalism implies that life is independent of its material substrate. For example, certain types of self-organizing computer programs can exhibit life-like functions, and "artificial life" proponents view such systems as "alive."53 Functionalists also point out that life's material substrate doesn't distinguish biological matter. Proteins, DNA, carbohydrates, fats, and other biomolecular components are made of the same atoms and elements that make up inanimate substances. Bolstered by the success of genetic engineering, functional/reductive approaches dominate molecular biology. "Life" is ascribed to an emergent property of biochemical processes.

Nonetheless, a commonly held contrary viewpoint is that functional descriptions fail to capture an essential selforganizing "unitary oneness" present in living systems. To nineteenth-century biologists this quality was ascribed to a "life force," "elan vital," or energy field. Then, as molecular and cell biology began to reveal the biochemical and physical processes involved in cellular activities, the apparent need for a life force waned, and "vitalists" (or "animists") were vilified. In modern reductionist science the notion of a life force, energy or information field has remained almost taboo. However, nineteenth-century vitalism was based either completely on electromagnetism or on forces outside the realm of science. Quantum effects were unknown.

There are three types of theories regarding the origin of life. One involves a "Creator", the second involves spontaneous chemical activity leading to biomolecules, and the third involves seeding of the earth from extraterrestrial sources. The 
extraterrestrial theory (e.g. carried by meteorites) is supported by the timing of life's evolutionary development on earth, but still requires an explanation for life's origin somewhere else. ${ }^{54}$

Peptides, simple sugars, lipids and nucleic acid could have formed spontaneously in salt water, for example in tide pools or near thermal vents, or in clay minerals. ${ }^{55}$ The big mystery is how these simple biomolecular precursors evolved to organelles and cells.

Proteins, lipids and nucleic acids are composed of constituent molecules which have both non-polar and polar regions on opposite ends. In an aqueous medium the non-polar regions of any of these components will join together to form hydrophobic regions where quantum forces reign. Perhaps the first forms of life were tiny oil-like pockets-shielded from the aqueous environment-in which quantum interactions organized, linked together and regulated increasingly larger biomolecules. As non-polar aromatic groups absorb and are excited by photons, organized arrays of such groups (i.e. cytoskeletal structures) may have induced cytoplasm to evolve as phase-ordered matter. ${ }^{56}$

In cytoplasm, cytoskeletal structures and ordered water on their surfaces are ideal media for quantum coherence as suggested by Fröhlich, able to utilize nonspecific metabolic energy and heat to promote coherence (rather than decoherence), and possibly generate, perceive and trap evanescent photons.

The evolutionary origin of centrioles, cilia and flagella (which have the same basic structure of nine microtubule doublets or triplets arranged in a larger cylinder, but with additional motor proteins) is unclear. According to the endosymbiotic theory, ${ }^{57}$ our eukaryotic cells arose from symbiosis, an invasion of simple bacteria-like prokaryotes by mitochondria which supplied energy, and by flagellates (e.g. spirochetes) which brought cytoskeletal proteins providing structural support, compartmentalization and internal organization, movement and perhaps intelligence and eventually consciousness. The origin of flagellates is unknown.

There is some question as to whether centrioles, cilia and flagella (i.e. flagellates) could have evolved purely by natural selection, as they are said to exhibit "irreducible complexity". ${ }^{58}$ Darwin said in The Origin of Species:

"If it could be demonstrated that any complex organ existed which could not possibly have been formed by numerous, successive, slight modifications, my theory would absolutely break down."

Innumerable small, randomly chosen steps of incremental changes in proteins to form tubulin, and tubulin to form microtubules, and microtubules to form centrioles, cilia and flagella would seem to offer no advantages "along the way". Consequently centrioles, cilia and flagella have been suggested as examples of "intelligent design". ${ }^{88}$ Designed by what, or by whom? This question leads some to "Creationism". But there is also the view that intelligent design reflects the type of Platonic information embedded in the Planck scale suggested by Roger Penrose. ${ }^{59,60}$ If so, then via quantum states living systems are in touch with a deeper reality. Does this imply that quantum information devices, for example, would also be "alive"? Not necessarily, as only organic molecules and cytoskeletal protein lattices may have the inherent flexibility to harness ambient energy for quantum coherent states, interact with the Planck scale via quantum gravity processes, and utilize photons as phase-ordered matter.

\section{Acknowledgements}

We thank Dave Cantrell for illustrations and Patti Bergin for manuscript preparation

\section{References}

1. E. Schrodinger, What is life? Cambridge University Press, Cambridge UK, 1944.

2. P. Davies. "Physics and life, in the first steps of life in the Universe", Proceedings of the Sixth Trieste Conference on Chemical Evolution, Editors: J. Chela-Flores, Owen, Tobias and F. Raulin, Academic Publishers, Dordrecht, The Netherlands, 18-22 September 2001.

3. S.R. Hameroff. "Quantum vitalism”, Advances: The Journal of Mind-Body Health , 13(4), 13-22, 1997.

4. S. Hagan, S.Hameroff, J. Tuszynski,. "Quantum computation in brain microtubules? Decoherence and biological feasibility", Physical Reviews E, 65, 061901, 2002.

5. S. Hameroff "Anesthesia, consciousness and hydrophobic pockets" Toxicology Letters 100/101:31-39, 1998.

6. D. Voet, J.G. Voet, J.G. Biochemistry, Wiley, 2nd edition, New York, 1995. 
7. A. Bairoch and R. Apweiler. "The swiss-prot protein sequence data bank and its supplement TrEMBL", Nucl. Acids Res., 26, 38-42, 1998.

8. E. Nogales, S.G. Wolf, K.H. Downing. "Structure of the tubulin dimer by electron crystallography". Nature 391, 199-203, 1998.

9. M. Karplus, J.A. McCammon. "Protein ion channels, gates, receptors". In. Dynamics of Proteins: Elements and Function, Ann. Rev. Biochem., J. King (ed.), Benjamin/Cummings, Menlo Park, 40, 263-300, 1983.

10. Y. Jiang, V. Ruta, J. Chen, A. Lee, R. MacKinnon. "The principle of gating charge movement in a voltagedependent K+ channel". Nature, 423(6935), 42-8, 2003.

11. S.R. Hameroff, J. Tuszynski.. "Search for quantum and classical modes of information processing in microtubules: implications for the living state", In: Bioenergetic Organization in Living Systems, Eds. Franco Musumeci, Mae-Wan Ho, World Scientific, Singapore. 2003.

12. M. Conrad. "Amplification of superpositional effects through electronic conformational interactions". Chaos, Solitons and Fractals, 4, 423-438. 1994.

13. N. Hirokawa. "Molecular architecture and dynamics of the neuronal cytoskeleton". In: The neuronal cytoskeleton, Ed. R. Burgoyne. New York, Wiley-Liss, pp. 5-74, 1991

14. A. Roitberg, R.B Gerber, R.R. Elber, M.A. Ratner. "Anharmonic wave functions of proteins: quantum self consistent field calculations of BPTI". Science, 268(5315), 1319-1322, 1995.

15. J. Tejada, S. Garg, D.D. Gider, Awschalom, D. DiVincenzo, D.Loss. "Does macroscopic quantum coherence occur in ferritin?" Science, 272, 424-426, 1996.

16. J. Klein-Seetharaman, M. Oikawa, S.B. Grimshaw, J. Wirmer, E. Duchardt, T. Ueda, T. Imoto, L.J. Smith, C.M. Dobson, H. Schwalbe. "Long-range interactions within a nonnative protein". Science, 295, 1719-1722, 2002.

17. J. Satinover. The quantum brain, Wiley and Sons, New York, 2001.

18. H. Frauenfelder, P.W. Fenimore, B.H. McMahon "Hydration, slaving and protein function" Biophysical Chemistry. 98(1-2):35-48, 2002

19. G.H. Pollack. Cells, gels and the engines of life, Ebner and Sons, Seattle, 2001.

20. E. Karsenti, I. Vernos. "The mitotic spindle: A self-made machine". Science 294, 543-547, 2001.

21. P. Dustin. Microtubules, 2nd Revised Ed., Berlin: Springer, 1984.

22. J. Tuszynski, S. Hameroff, M.V. Sataric, B. Trpisova, M.L.A. Nip. "Ferroelectric behavior in microtubule dipole lattices; implications for information processing, signaling and assembly/disassembly". J. Theor. Biol. 174, 371-380, 1995

23. H. Fröhlich. "Long-range coherence and energy storage in biological systems". Int. J.Quantum Chem. 2, 6419, 1968.

24. H. Fröhlich. "Long-range coherence and the actions of enzymes". Nature. 228, 1093, 1970.

25. H. Fröhlich. "The extraordinary dielectric properties of biological materials and the action of enzymes". Proc. Natl. Acad. Sci. 72, 4211-4215, 1975.

26. A. Samsonovich, A.C. Scott, S.R. Hameroff. "Acousto-conformational transitions in cytoskeletal microtubules: Implications for information processing". Nanobiology, 1, 457-468, 1992.

27. G. Albrecht-Buehler. "Rudimentary form of cellular "vision", Proc. Natl Acad Sci. USA, 89(17), 8288-8292, 1992.

28. S. Hameroff "A new theory of cancer: Centrioles, mitosis and quantum entanglement" submitted

29. M. Jibu, S. Hagan, K. Pribram, S.R. Hameroff, K. Yasue. "Quantum optical coherence in cytoskeletal microtubules: implications for brain function”. BioSystems, 32:195-209, 1994.

30. L. Lauck, A.R. Vasconcellos, R Luzzi. “On Fröhlich's coherent effects in biological systems: Influence of carriers and high order dissipative effects". J Theor Biol, 158, 1-13, 1992

31. W. Grundler, and F. Keilmann. "Sharp Resonances in Yeast Growth Proved Nonthermal Sensitivity to Microwaves". Phys. Rev. Letts., 51, 1214-1216, 1983.

32. E. Pessa, G. Vitiello. "Quantum dissipation and neural net dynamics". Bioelectrochemistry \& Bioenergetics, 48(2), 339-42, 1999.

33. BL.Reid, C. Bourke. "Attempts to identify a control system for chemical reactivity in the living state using virtual energy". Medical Hypotheses, 57(1), 6-22, 2001.

34. S. Celaschi, S. Mascarenhas. "Thermal Stimulated Pressure and Current Studies of Bound Water in Lysozyme". Biophys. J., 29(2), 273-277, 1977.

34. E. Del Giudice. "Collective Properties of Biological Systems". In: Modern Bioelectrochemistry. F. Gutmann and H. Keyzer eds., New York, Plenum Press, 1986. 
35. E. Del Giudice, S. Doglia, M. Milani, "Self Focusing of Fröhlich Waves and Cytoskeleton Dynamics", Phys. Letts, 90A, 104-106, 1982.

36. E. Del Giudice, S. Doglia, M. Milani, G. Vitiello. "Spontaneous Symmetry Breakdown and Boson Condensation in Biology”. Phys. Rev. Letts., 95(a), 508-510, 1983.

37. M Jibu, S. Hagan, S.R. Hameroff, K.H. Pribram, K.Yasue. "Quantum optical coherence in cytoskeletal microtubules: implications for brain function". BioSystems, 32, 195-209, 1994.

38. M. Jibu, K. Yasue, K. Quantum brain dynamics: an introduction. John Benjamins, Amsterdam, 1995.

39. S.K. Lamoreaux. "Demonstration of the Casimir force in the 0.6 to 6 micron range". Physical Review Letters, 78(1), 5-8, 1997.

40. G.L. Hall. "Quantum electrodynamic (QED) fluctuations in various models of neuronal microtubules". Consciousness Research Abstracts -Tucson II (Journal of Consciousness Studies) Abstract 145, 1996.

41. R. Penrose, S. Hameroff. "Gaps, what gaps?" Reply to Grush and Churchland. Journal of Consciousness Studies, 2(2), 99-112, 1995.

42. S.R. Hameroff, R. Penrose. "Orchestrated reduction of quantum coherence in brain microtubules: A model for consciousness", in S.R. Hameroff, A. Kaszniak, and A.C. Scott (eds.), In: Toward a Science of Consciousness The First Tucson Discussions and Debates, MIT Press, Cambridge, MA. Also published in: Mathematics and Computers in Simulation, 40, 453-480, 1996.

43. S.R. Hameroff, and R. Penrose. "Conscious events as orchestrated spacetime selections". Journal of Consciousness Studies, 3(1), 36-53, 1996.

44. S.R. Hameroff. "Quantum computation in brain microtubules? The Penrose-Hameroff 'Orch OR' model of consciousness". Philosophical Transactions Royal Society, London (A) 356, 1869-1896, 1998.

45. M. Ouyang, D.D. Awschalom. "Coherent spin transfer between molecularly bridged quantum dots". Science, 301, 1074-1078, 2003.

46. A. Grinvald, A. Arieli , M. Tsodyks, T. Kenet. "Neuronal assemblies: single cortical neurons are obedient members of a huge orchestra”. Biopolymers, 68(3), 422-36, 2003.

48. M. Tegmark. "The importance of quantum decoherence in brain processes". Phys. Rev. E, 61, 4194-4206, 2000.

49. A. M. Steane. "Introduction to quantum error correction". Phil. Trans. Roy. Soc. Lond, A, 356, 1739-1758.

50. M.H. Freedman, A. Kitaev, M.J. Larsen, Z. Wang.“Topological quantum error correction”. quant-ph/0101025, 2002.

51. S. Hameroff, A. Nip, M. Porter, J. Tuszynski. "Conduction pathways in microtubules, biological quantum computation, and consciousness". BioSystems, 64,149-168, 2002.

52. V.N. Binhi, A.V. Savin. "Molecular gyroscopes and biological effects of weak extremely low-frequency magnetic fields". Phys Rev E, 65: 051912-1\&8211:051912-10, 2002.

53. S. Hameroff, S. Rasmussen, B. Mansson. "Molecular automata in microtubules: basic computational logic of the living state". In Artifical Life: SFI Studies in the Science of Complexity, ed. C. Langton, Addison-Wesley, New York, pp. 521-553.

54. M.A. Line. "The enigma of life and its timing"? Microbiology, 148, 21-27, 2002.

55. A.G. Cairns-Smith Clay Minerals and the Origin of Life, Cambridge, Cambridge University Press, 1986.

56. M.O. Scully. "Light at a standstill?". Nature, 426, 610-611, 2003.

57. L. Margulis. Origin of Eukaryotic Cells. New Haven, Yale University Press, 1975.

58. S. White, W. Meier, F. Lovell, A. McCoy, C.J. Robinove, T.F. Creelan, R.Brun, I. Gordon, R. MacWest, I.E. Collier, D.T. Gish, W.K. Hartmann, M.J. Behe. "Educators have a hard choice nationally, not just in Kansas". Science. 289(5481), 869-71, 2000

59. R.Penrose. The Emperor's New Mind, Oxford Press, Oxford, U.K. 1989.

60. R. Penrose. Shadows of the Mind, Oxford Press, Oxford, U.K.1994. 